Cite this: Phys. Chem. Chem. Phys., 2013, 15, 3772

Received 7th November 2012, Accepted 10th January 2013

DOI: $10.1039 / c 3 c p 43968 b$

www.rsc.org/pccp

\section{The catalytic mechanism of glyceraldehyde 3-phosphate dehydrogenase from Trypanosoma cruzi elucidated via the QM/MM approach $†$}

\author{
Mauro Reis, ${ }^{a b}$ Cláudio Nahum Alves, ${ }^{\text {a }}$ Jerônimo Lameira, ${ }^{a}$ Iñaki Tuñón, ${ }^{b}$ \\ Sergio Martí ${ }^{\mathrm{c}}$ and Vicent Moliner*c
}

Glyceraldehyde-3-phosphate dehydrogenase (GAPDH) has been identified as a key enzyme involved in glycolysis processes for energy production in the Trypanosoma cruzi parasite. This enzyme catalyses the oxidative phosphorylation of glyceraldehyde 3-phosphate (G3P) in the presence of inorganic phosphate (Pi) and nicotinamide adenosine dinucleotide (NAD+). The catalytic mechanism used by GAPDH has been intensively investigated. However, the individual roles of $\mathrm{Pi}$ and the $\mathrm{C} 3$ phosphate of G3P (Ps) sites, as well as some residues such as His194 in the catalytic mechanism, remain unclear. In this study, we have employed Molecular Dynamics (MD) simulations within hybrid quantum mechanical/molecular mechanical (QM/MM) potentials to obtain the Potential of Mean Force of the catalytic oxidative phosphorylation mechanism of the G3P substrate used by GAPDH. According to our results, the first stage of the reaction (oxidoreduction) takes place in the Pi site (energetically more favourable), with the formation of oxyanion thiohemiacetal and thioacylenzyme intermediates without acid-base assistance of His194. Analysis of the interaction energy by residues shows that Arg249 has an important role in the ability of the enzyme to bind the G3P substrate, which interacts with NAD+ and other important residues, such as Cys166, Glu109, Thr167, Ser247 and Thr226, in the GAPDH active site. Finally, the inhibition mechanism of the GAPDH enzyme by the 3-(p-nitrophenoxycarboxyl)-3-ethylene propyl dihydroxyphosphonate inhibitor was investigated in order to contribute to the design of new inhibitors of GAPDH from Trypanosoma cruzi.

\section{Introduction}

Chagas disease (American trypanosomiasis) is a protozoan infection caused by the flagellate Trypanosoma cruzi, ${ }^{1}$ which is endemic in 21 countries across Latin America. Chagas disease causes more fatalities in the region each year than any other parasite-borne disease, including malaria. According to the Drugs for Neglected Diseases initiative $(2010)^{2}$ there are about 8 million cases, 14000 deaths annually, and 667000

\footnotetext{
${ }^{a}$ Laboratório de Planejamento e Desenvolvimento de Fármacos, Instituto de Ciências Exatas e Naturais, Universidade Federal do Pará, CP 11101, 66075-110, Belém, PA, Brazil. E-mail: nahum@ufpa.br; Fax: +55 91-32017633; Tel: +55 91-32018235

${ }^{b}$ Departament de Química Física, Universitat de València, 46100 Burjassot, València, Spain

${ }^{c}$ Departament de Química Física i Analitica, Universitat Jaume I, 12071 Castellón, Spain. E-mail: moliner@uji.es; Fax: +34 964-728066; Tel: +34 964-728084 $\dagger$ Electronic supplementary information (ESI) available. See DOI: 10.1039/ c3cp43968b
}

disability-adjusted life years (DALYs). Chronic Chagas disease results in significant disability with great social and economic impact including unemployment and decreased earning ability. In Brazil, losses of over \$US 1.3 billion in wages and industrial productivity were due to workers with Chagas disease. Patient numbers are growing in non-endemic, developed countries (e.g., Australia, Canada, Japan, Spain and United States) due to increased migration of Latin American citizens unknowingly carrying the parasite in their blood.

Although discovered in 1909 by the Brazilian sanitary doctor Carlos Chagas, the disease still has no effective treatment; the drugs most commonly used to combat the disease, nifurtimox and benznidazole, are not very efficient and cause strong side effects. ${ }^{3}$ In search of targets more efficient and more specific for the disease, Opperdoes and Borst $(1977)^{4}$ found that the bloodstream from parasites of the Trypanosomatidae family possesses a microbody like organelle called a glycosome in which glycolysis occurs. Owing to the absence of functional mitochondria, the bloodstream of Trypanosoma brucei is 
entirely dependent on glycolysis for its energy production. ${ }^{5}$ Biochemical studies indicate that the Trypanosoma cruzi axenic amastigote intracellular form is highly dependent on the catabolism of carbohydrates as an energy source. ${ }^{6}$ The high dependence on glycolysis for energy production has made this metabolic pathway and the enzymes involved as promising targets for the design of anti-trypanosome drugs. Bakker and co-workers ${ }^{7}$ identified glyceraldehyde-3-phosphate dehydrogenase (GAPDH), phosphoglycerate kinase (PGK) and fructose-1,6bisphosphate aldolase (ALD) as key enzymes involved in controlling the glycolytic flux of the parasite. Moreover, studies on erythrocyte glycolysis concluded that a 95\% deficiency in these enzymes does not cause any clinical symptoms. ${ }^{7}$ The inhibition of GAPDH leads to a significant reduction of glycolysis in the parasite. ${ }^{7,8}$ The mechanism of the homotetrameric GAPDH has been well studied. ${ }^{9-19}$ GAPDH catalyses the oxidative phosphorylation of glyceraldehyde 3-phosphate (G3P) to 1,3bisphosphoglycerate (1,3-BPG) using nicotinamide adenosine dinucleotide (NAD+) and inorganic phosphate (Pi). Experimental kinetic studies published by Michels et al. (1996) show evidence of the formation of the product 1,3-BPG. However, there is no general agreement on the molecular mechanism. Schemes 1 and 2 show three possible mechanisms for the oxidoreduction stage used by GAPDH involving the G3P substrate. In the first proposed mechanism depicted in Scheme 1A, the G3P substrate adopts a cis conformation and first binds to the Ps site of the GAPDH active site, where the reaction is started with a nucleophilic attack of the Cys166 thiolate function on the aldehydic carbon C1 of the G3P substrate, which leads to the formation of a tetrahedron intermediate (hemithioacetal, HTA). A hydride transfer assisted by His194 follows this step. In the next step, a conformational change in the substrate is necessary to allow for $\mathrm{NADH}$ release, while the $\mathrm{C} 3 \mathrm{P}$ group of the substrate flips toward the new Pi site. ${ }^{21,22}$ In the second proposed mechanism depicted in Scheme 1B, the G3P substrate adopts a cis conformation, however, this step takes place without the acid-base assistance of His194. Finally, in the third proposed mechanism in Scheme 2, the substrate G3P adopts the trans conformation, where the G3P substrate first binds to the Pi site in the oxidoreduction stage (see Schemes $1 \mathrm{~B}$ and 2) and then flips from Pi to the Ps site in the phosphorylation stage. ${ }^{18-20,23}$ Recently, crystallographic studies conducted by Castilho et al. $(2003)^{23}$ reported the refined glycosomal Trypanosoma cruzi GAPDH in complex with an analogue of the thioacyl intermediate. Their results are consistent with the experimental studies of Corbier et al. $(1994)^{20}$ and Yun, Park, Kim and Park (2000), ${ }^{24}$ which give strong experimental support for the generally accepted flip-flop model of the catalytic mechanism in GAPDH.

A common feature of these structures is the presence of two highly conserved cationic sites, which were assumed to be the two phosphate binding sites; Ps is the C3 phosphate of G3P, and $\mathrm{Pi}$ is the inorganic phosphate that were identified in the GAPDH active site. The location of the Ps site in the threedimensional structure is conserved and independent of the enzyme state. This Ps site is composed of the side chains of residues Thr199, Thr197 and Arg249 and the 2-hydroxyl group of the nicotinamide ribose of NAD (Fig. 1). On the other hand, the location of the Pi site appears to vary depending on the

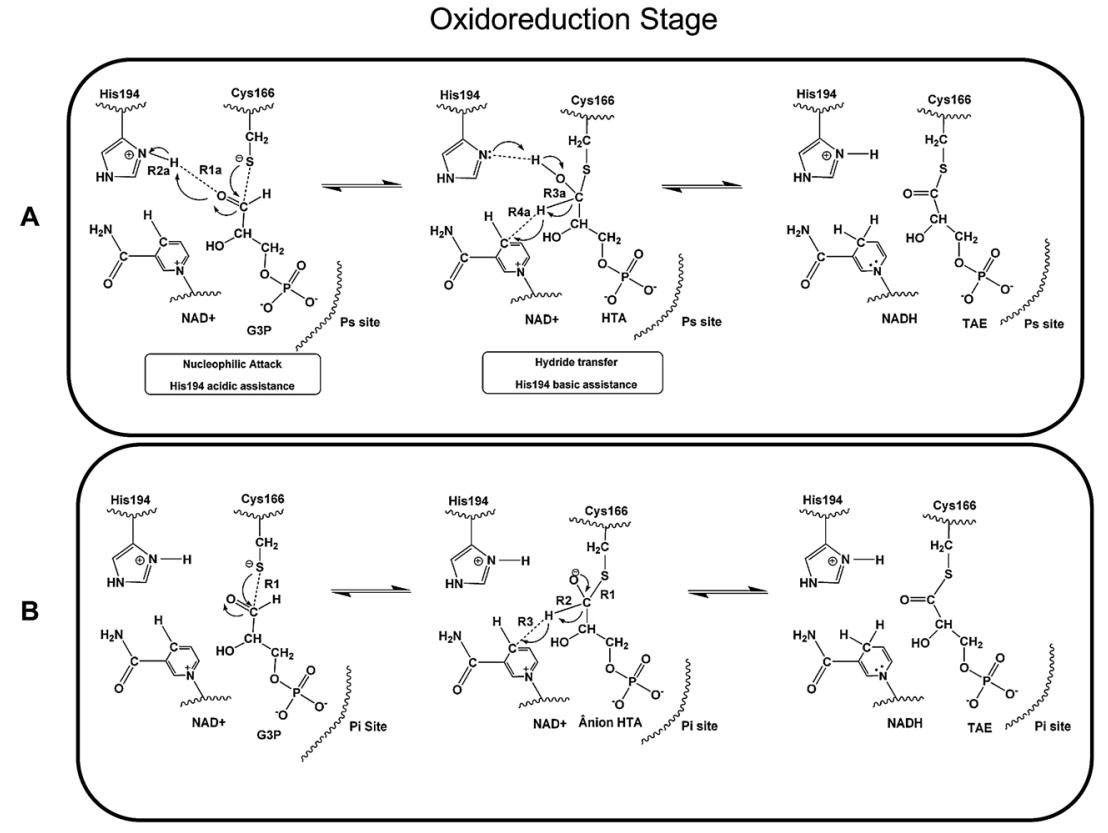

Scheme 1 Two possible reaction pathways for the oxidoreduction stage of Trypanosoma cruzi GAPDH. (A) The C3 phosphate first interacts with the Ps site. The hydride transfer occurs through acid/base catalysis by His194 (see ref. 21 and 22). (B) The C3 phosphate first interacts with the Pi site. The hydride transfer occurs without the assistance of His194 (see ref. 18-20 and 23). For both A and B, the reaction begins with a nucleophilic attack of the Cys 166 thiolate function on the aldehydic carbon C1 of the G3P substrate. This leads to tetrahedron intermediate formation of hemithioacetal (HTA) (A) or anion hemithioacetal (B) and then the thioacylenzyme (TAE). 

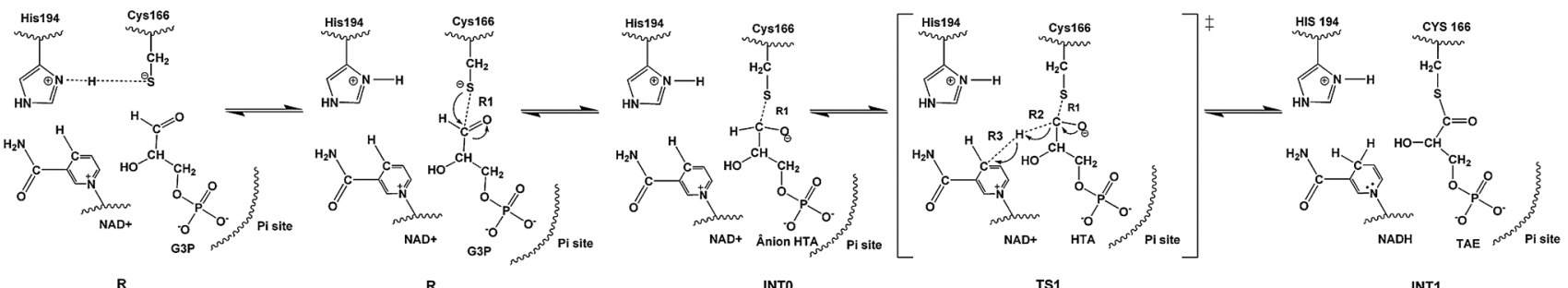

G3P

INTO

TS1
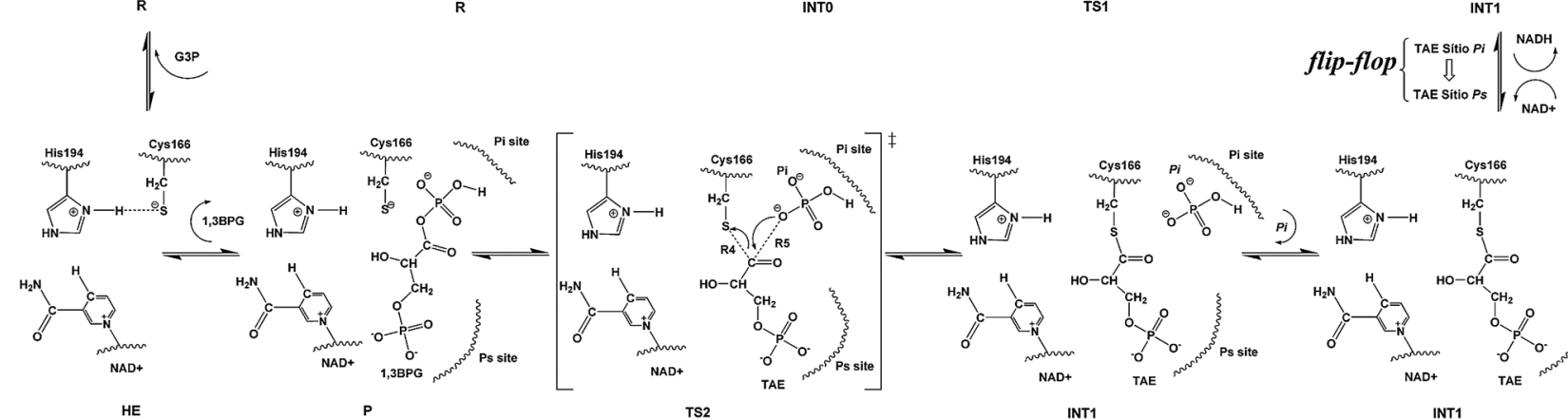

TS2

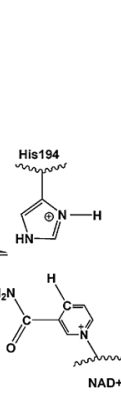

flip-flop $\{\begin{array}{c}\text { TAE Sitio } P i \\ \prod \\ \text { TAE Strio Ps }\end{array} \mid \overbrace{\text { NAD+ }}^{\text {NADH }}$

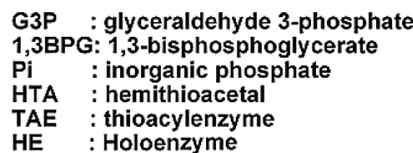

Scheme 2 Proposed catalytic mechanism.

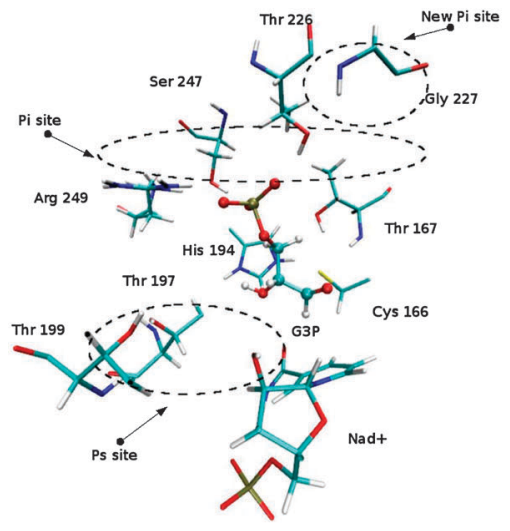

A

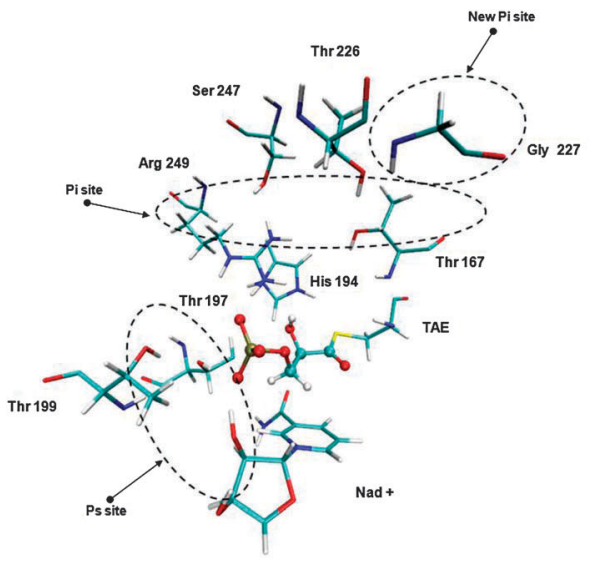

B

Fig. 1 The location of the Pi and Ps sites in the three-dimensional structures of Trypanosoma cruzi GAPDH.

presence and the nature of the bound ligands or source organisms. Although the most common conformation is that originally found in the holoenzyme from $H$. americanus and Bacillus stearothermophilus. ${ }^{15,18}$ An alternative conformation was first observed in the holoenzyme from Thermotoga maritima and Leishmania mexicana ${ }^{25,26}$ that generates a Pi site located closer to the catalytic Cys149 residue and $3.0 \AA$ away from the former position, which has been called a new Pi site. Castilho et al. $(2003)^{23}$ reported a new location for the site Pi in the GAPDH of Trypanosoma cruzi, where the phosphate group of a G3P analogue forms hydrogen bonds with residues Thr226 and Arg249. In this conformation, the phosphonate group is $3.35 \AA$ to the position previously described for the site of the phosphate in GAPDH L. mexicana ${ }^{26}$ and $4.81 \AA$ A to the position described in Trypanosoma brucei (Vellieux, Hajdu, and Hol, 1995). ${ }^{27}$ Ladame et al. $(2003)^{28}$ reported a new location for the Pi site in GAPDH in T. cruzi. This new binding site is very close to that previously identified in the 3D structure of GAPDH in T. cruzi reported by Castilho et al. (2003). ${ }^{23}$ Thus, we must consider the different locations of the binding sites for Pi, which can be dependent on the organism. In our particular case, we will consider these characteristics of $T$. cruzi GAPDH (Fig. 1). 
Molecular Dynamics (MD) simulations with hybrid quantum mechanics/molecular mechanics (QM/MM) potentials have been previously performed to study enzymatic catalytic mechanisms and protein-inhibitor interactions..$^{29-33}$ In the QM/MM method, a small part of the system (ligand/substrate species) is described by quantum mechanics, while the protein and solvent environment is represented by MM force fields. ${ }^{34,35}$ Recent reviews have addressed the use of the QM/MM approach for biomolecular systems with an emphasis on enzymatic reactions. $^{35-38} \mathrm{QM} / \mathrm{MM}$ methods have also been used in drug design. $^{36-39}$ Recently, hybrid QM/MM methods have been successfully employed to determine the first step of the catalytic mechanism used by $O$-GlcNAcase involving substrate-assisted catalysis. $^{30}$ More recently, we have studied the inhibition mechanism of GAPDH involving an iodoacetate (IAA) inhibitor using the hybrid QM/MM MD. ${ }^{40}$

The analysis of free energy profiles has been performed to gain insight into the pathway of the reaction and provide theoretical support for the generally accepted catalytic mechanism. ${ }^{29-33}$ Potential of Mean Force (PMF) from biased MD simulations using an adequate distinguished reaction coordinate may be employed to get the free energy profile of the transformation from reactants to products. ${ }^{37}$ In this report, we have investigated the catalytic mechanism used by GAPDH of $T$. cruzi involving oxidative phosphorylation of G3P to 1,3-BPG (Schemes 1 and 2) from free energy profiles obtained in terms of two-dimensional PMFs (2D PMFs) using QM/MM potentials. Particular attention has been paid to the role of His194 in this mechanism and the main site (Pi or Ps?) where the reaction begins. In addition, we have also investigated the inhibition mechanism of the GAPDH enzyme by the 3-( $p$-nitrophenoxycarboxyl)-3-ethylene propyl dihydroxyphosphonate inhibitor in order to understand the inhibition mechanism and contribute to the design of new inhibitors. Finally, analysis of interaction energies between the substrate (reactants, TS and intermediate), cofactor, and protein was performed in order to determine the role of key residues during the reaction.

\section{Methods}

\section{The system}

The crystal structures of GAPDH have been determined at different resolution from various organisms, among them American lobster, ${ }^{15,49}$ humans, ${ }^{41,42}$ Bacillus stearothermophilus, ${ }^{18,22,50}$ Thermotoga maritima, ${ }^{25}$ Thermus aquaticus, ${ }^{43}$ Escherichia coli, ${ }^{44}$ Leishmania mexicana, ${ }^{26,45}$ Trypanosoma brucei $i^{27,46}$ and $T$. cruzi. $^{23,47,48}$ In this study, the initial coordinates for computational simulations were taken from the tetrameric crystal structure of the T. cruzi GAPDH complex at $1.95 \AA$ resolution (PDB code $1 \mathrm{~K} 3 \mathrm{~T}){ }^{48}$ All simulations were done using the fDynamo library. ${ }^{51,52}$

The standard $\mathrm{p} K_{\mathrm{a}}$ values of ionizable groups can be shifted by local protein environments. ${ }^{53}$ Therefore, an accurate assignment of the protonation states of all these residues at $\mathrm{pH} 7$ was carried out by recalculating the standard $\mathrm{p} K_{\mathrm{a}}$ values of the titratable amino acids using the empirical propKa program of Jensen and co-workers (2005). ${ }^{54}$ According to this result, most of all residues were found in their standard protonation state. On the other hand, Polgar has suggested the existence of a His $194^{+} / \mathrm{Cys} 166^{-}$ion pair, ${ }^{66}$ where the $\mathrm{p} K_{\mathrm{a}}$ corresponds to 8.2 and 5.5 for Cys166 and His194, respectively. ${ }^{66}$ Additionally, under physiological conditions, a Cys166 residue shows considerable nucleophilic reactivity in the active site of GAPDH. In order to determine the existence of the His $194^{+} / \mathrm{Cys} 166^{-}$ ion-pair, we have obtained a potential energy surface (PES) using two coordinates that describe the proton transference from Cys166 to His194 (Fig. S1, ESI $\dagger$ ). The result shows that the formation of the His $194^{+} / \mathrm{Cys} 166^{-}$ion-pair occurs spontaneously, which is consistent with the results of Cartier and co-workers. ${ }^{55}$

After adding the hydrogen atoms to the structure, a series of optimization algorithms [steepest descent conjugated gradient and L-BFGS-B $]^{56}$ were applied. To avoid a denaturation of the protein structure, all the heavy atoms of the protein and the inhibitor were restrained by means of a Cartesian harmonic umbrella with a force constant of $1000 \mathrm{~kJ} \mathrm{~mol}^{-1} \AA^{-2}$. Afterwards, the system was fully relaxed, but the peptidic backbone was restrained with a lower constant of $100 \mathrm{~kJ} \mathrm{~mol}^{-1} \AA^{-2}$. The optimised protein was solvated with a water molecules sphere of $80 \AA$ radius using the principal axis of the protein-substrate complex as the geometrical centre. Any water with an oxygen atom lying in a radius of $2.8 \AA$ from a heavy atom of the protein was deleted. The remaining water molecules were then relaxed using optimisation algorithms. Finally, 100 ps of hybrid QM/ MM Langevin-Verlet MD (NVT) at $300 \mathrm{~K}$ was used to equilibrate the model. During MD, the atoms of the substrate, the nicotinamide ring and the adjacent ribose of NAD+ and a side chain of $\mathrm{His} 194^{+}$and $\mathrm{Cys} 166^{-}$residues (present in the active site) were selected for treatment by QM, using the semiempirical AM1 Hamiltonian, ${ }^{57}$ resulting in 59 atoms in the QM region. The rest of the system (protein plus water molecules) was described using the OPLS-AA ${ }^{58}$ and TIP3 ${ }^{59}$ force fields, respectively, as implemented in the fDynamo library. ${ }^{51,52}$ To saturate the valence of the QM/MM frontier, we used the link atoms procedure, placing these link atoms between the $\mathrm{C}^{\alpha}$ and $\mathrm{C}^{\beta}$ atoms of the His $194^{+}$and $\mathrm{Cys} 166^{-}$residues, and between the $\mathrm{C}^{\prime}{ }^{\prime}$ and $\mathrm{C}^{\prime}$ atoms of the ribose ring of NAD+. The final system contains a total of 39546 atoms. Due to the amount of degrees of freedom, any residue $18 \AA$ apart from any of the atoms of the initial inhibitor was selected to be kept frozen in the remaining calculations. Cut-offs for the nonbonding interactions were applied using a switching scheme within a range of radius of 14.5 to $18 \AA$ A. Afterwards, the system was equilibrated by means of $600 \mathrm{ps}$ of QM/MM MD at a temperature of $300 \mathrm{~K}$. The computed RMSD for the protein renders a value always below $0.03 \AA$. Furthermore, the RMS of the temperature along the different equilibration steps was always lower than $2.5 \mathrm{~K}$, and the variation coefficient of the potential energy during the dynamics simulations was never higher than $0.4 \%$.

The potential energy surface (PES). The fDynamo library ${ }^{51,52}$ was used to explore different PES as a function of interatomic distances. In order to verify the mechanism for the oxidoreduction 
stage proposed by Moniot et al. (2008), ${ }^{22}$ two antisymmetric combinations of distances $\mathrm{R} 1 \mathrm{a}-\mathrm{R} 2 \mathrm{a}$ and $\mathrm{R} 3 \mathrm{a}-\mathrm{R} 4 \mathrm{a}$ were used as distinguished reaction coordinates to generate the PES (see Scheme 1A). G3P adopts a cis conformation and initially binds to the active site with $\mathrm{C} 3$ phosphate in the Ps site, and R1a-R2a corresponds to general acid-base catalysis by His194 at G3P and R3a-R4a corresponds to coordinates hydride transfer to NAD+. Another possibility was also studied with the intention of verifying the participation of His194 in the oxidoreduction stage. In this case, the PES was built with the R1 and R2-R3 distances (see Schemes $1 \mathrm{~B}$ and 2 oxidoreduction stage), where the substrate is initially bound to the active site with the group C3P in the site Pi and where the G3P can adopt two different conformations (cis and trans). In the cis conformation (see Scheme 1B), the carbonyl of G3P points to the His194 and discards the coordinates of the acid-base assistance His194 but considers a possible electrostatic interaction of His194 with the carbonyl group of G3P. In the trans conformation (see Scheme 2), the carbonyl group of the G3P substrate points to the opposite side of the His194 preventing or hindering the assistance acid-base or electrostatic interaction of the carbonyl of G3P with this residue. R1 corresponds to the nucleophilic attack of Cys166 ${ }^{-}$on the $\mathrm{C} 1$ aldehydic group of G3P, and R2-R3 corresponds to hydride transfer from G3P to NAD+.

The Potential of Mean Force (PMF). In order to obtain the $\mathrm{PMF}^{60,61}$ associated with the oxidative phosphorylation reaction of $\mathrm{G} 3 \mathrm{P}$, the reaction coordinate was selected based on conclusions of the pathway described in the PES. All PMFs have been calculated using the weighted histogram analysis method (WHAM) combined with the umbrella sampling approach $^{62,63}$ as implemented in fDynamo. ${ }^{51,52}$

To estimate the activation free energy from this quantity we recovered one-dimensional PMF changes tracing a maximum probability reaction path on the 2D-PMF surface and integrating over the perpendicular coordinate (see ESI $\dagger$ and ref. 64 and 65 for more details). In constructing the 2D-PMF, the distinguished reaction coordinates were R1 and R2-R3, as defined in Scheme 2 (oxidoreduction stage). A total of 12 simulations were performed at different values of $\mathrm{R} 1$ (12 simulations in a range from +1.7 to $+3.5 \AA$ ), with an umbrella force constant of $2800 \mathrm{~kJ} \mathrm{~mol}^{-1} \mathrm{~A}^{-1}$; in addition, a total of 40 simulations were performed at different values of $\mathrm{R} 2-\mathrm{R} 3$ (40 simulations in a range from -2.0 to $+2.0 \AA$ ), also with an umbrella force constant of $2800 \mathrm{~kJ} \mathrm{~mol}^{-1} \mathrm{~A}^{-1}$. The values of the variables sampled during the simulations were then pieced together to construct a full distribution function from which the 2D-PMF was obtained. On each window, 5 ps of relaxation was followed by 10 ps of production with a time step of 0.5 fs due to the nature of the chemical step involving a hydride transfer.

The use of QM/MM calculations in the evaluation of the free energy is commonly restricted to semiempirical Hamiltonians due to the large amount of gradient vector evaluations. One way to reduce the error associated with this quantum level of theory is the inclusion of correction terms in the potential energy function, particularly by means of interpolated corrections ${ }^{72}$ as follows:

$$
E=E_{\mathrm{AM} 1 / \mathrm{MM}}+S\left(\xi_{1}, \xi_{2}\right)
$$

where $S$ accounts for a two-dimensional derivable spline function, which is the result of the gas-phase difference between a high-level (HL) energy calculation for the QM subsystem and a low-level (LL) one. In the present work, the correction term accounts for the MP2/6-31G** level of theory (HL) and the semiempirical AM1 Hamiltonian (LL). These calculations were carried out using the Gamess-US (ver 11, 2008R1) program. ${ }^{73}$

\section{Results and discussion}

\section{Role for His194 in a catalytic mechanism}

A question not yet clarified is the role of the catalytic residue His (His176 in Escherichia coli, which is equivalent to His194 in T. cruzi) of GAPDH. The possible contribution of the His194 residue in the catalysis of GAPDH could be as: (1) a chemical activator of the Cys166 thiol group reactivity, (2) a base catalyst to favour the hydride transfer, and (3) a stabilizer of the tetrahedral transition states. ${ }^{21}$ Soukri et al. (1989) ${ }^{17}$ and Polgar $(1975)^{66}$ have proposed that the His176 in the active site of Escherichia coli GAPDH acts as a chemical activator. In a theoretical study, Cartier et al. (1999) ${ }^{55}$ have found a strong interaction through hydrogen bonding between the Cys149 ${ }^{-}$/ His176 ${ }^{+}$ion pair (Cys149 in Escherichia coli, which is equivalent to Cys166 in T. cruzi). This ion pair could explain the deep loss in activity when the Cys149 was replaced by Ser149, which is always in neutral form. ${ }^{55}$ Some mutagenesis studies have shown that His176 could act as a base in the hydride transference from the tetrahedron transition state. ${ }^{15,17,21}$ The existence of the Cys149 ${ }^{-} / \mathrm{His} 176^{+}$ion pair was validated through experimental assays. ${ }^{17,21,66}$ A molecular mechanism describing the oxidoreduction stage was presented by Moniot et al. (2008), ${ }^{22}$ where the C3P group of glyceraldehyde-3-phosphate could be localised in the Ps site (see Scheme 1A). The proposed reaction starts with an attack of Cys166 on the $\mathrm{C} 1$ atom of the substrate, which leads to tetrahedron intermediate (hemithioacetal, HTA) formation. The second step is assisted by His176, which acts as a base where the hydride transference from the $\mathrm{C} 1$ atom of the substrate to the $\mathrm{C} 4$ of the nicotinamide group of $\mathrm{NAD}+$ takes place (Scheme 1A). Then, in agreement with results reported by Moniot et al. (2008) $)^{22}$ the function of His176 is in a general base catalysis. In this study the catalytic residues His194 and Cys166 of $T$. cruzi are equivalent to residues His176 and Cys149 of E. coli and Bacillus strearothermophilus.

\section{Pi $\times$ Ps site}

The GAPDH enzyme has two anion recognition sites, Pi and Ps, ${ }^{15}$ and the main site where the catalytic mechanism could start remains unclear. ${ }^{22,23}$ Some mutagenesis studies were performed in order to clarify the molecular mechanism used by GAPDH. ${ }^{17,20,21}$ Skarzynski et al. $(1987)^{18}$ and Corbier et al. $(1994)^{20}$ have proposed that the C3P group of G3P would first interact with the $\mathrm{Pi}$ site. In the next stage, the coenzyme exchange probably leads to conformational isomerisation of the acyl group with the $\mathrm{C} 3$ phosphate flipping from the Pi to Ps 
site. On the other hand, Didierjean et al. (2003) ${ }^{67}$ exploring a mutation of Cys149 by Ala or Ser in Bacillus stearothermophilus GAPDH, provided conclusions that the reaction could initiate in the Ps site. Recently, Castilho et al. $(2003)^{23}$ studying T. cruzi GAPDH in complex with a covalently bound G3P analogue structure have proposed that the first stage of the reaction takes place in the $\mathrm{Pi}$ site. In addition, experimental results generally support the flip-flop model of the GAPDH catalytic mechanism, where the reaction initiates in the Pi site, then changes to the Ps site. ${ }^{18,20,24}$

\section{The PES}

The catalytic mechanism of GAPDH has been investigated through kinetics and crystallographic studies. ${ }^{18,20,22-24,67}$ Nevertheless, as mentioned, the individual role of Pi and Ps sites in the catalytic events remains unclear. In order to investigate the role of His194 in the first stage of the reaction and determine the site ( $\mathrm{Pi}$ or Ps) where the first stage of the catalytic mechanism used by GAPDH takes place, we have constructed potential energy surfaces (PESs) ${ }^{39}$ using AM1/MM and combining coordinates. The first PES (Fig. 2) was obtained through two antisymmetric combinations of distances R1a-R2a and R3a-R4a (see Scheme 1A), which correspond to the proton transference from the $\mathrm{N} 1$ atom of His194 to the $\mathrm{O} 1$ atom of glyceraldehyde 3-phosphate (G3P) and the hydride transference from the $\mathrm{C} 1$ atom of the hemithioacetal intermediate to the $\mathrm{C} 4$ atom of the nicotinamide group of $\mathrm{NAD}+$, respectively. The $\mathrm{R}$ on this PES (Fig. 2) corresponds to reactive G3P, the INT-M corresponds to the tetrahedron intermediate (HTA) assisted by His194 and the INT1 corresponds to the thioacylenzyme intermediate and NADH. This first PES (Fig. 2) was obtained with G3P in the Ps site showing two possible pathways; the first with the energy barrier of $105.47 \mathrm{~kJ} \mathrm{~mol}^{-1}$ did not lead to products, and the second with the energy barrier of $194.24 \mathrm{~kJ} \mathrm{~mol}^{-1}$ led to the expected intermediate INT1.

Two PES were built with symmetric and anti-symmetric combinations of the R1 and R2-R3 distances, where the substrate is initially bound to the active site with the group C3P in the site Pi and where the G3P can adopt two different conformations (cis and trans). The R1 corresponds to the nucleophilic attack of Cys166 ${ }^{-}$on the $\mathrm{C} 1$ aldehydic group of G3P, and R2-R3 corresponds to hydride transfer from G3P to NAD+. Fig. 3 shows the two surfaces obtained with the C3P group of $\mathrm{G} 3 \mathrm{P}$ in the $\mathrm{Pi}$ site and represented two possible conformations (cis and trans for G3P, respectively, see Fig. 3A and $\mathrm{B}$ ). We have estimated the energy barrier of the substrate in the cis conformation to be $143.5 \mathrm{~kJ} \mathrm{~mol}^{-1}$ while the barrier for the trans conformation would be $119.23 \mathrm{~kJ} \mathrm{~mol}^{-1}$. The energy values of the PES are summarized in the diagram shown in Fig. 4. In accordance with these results, the reaction takes place in the $\mathrm{Pi}$ site (energetically more favourable) in the trans conformation and the general base catalysis by His194 does

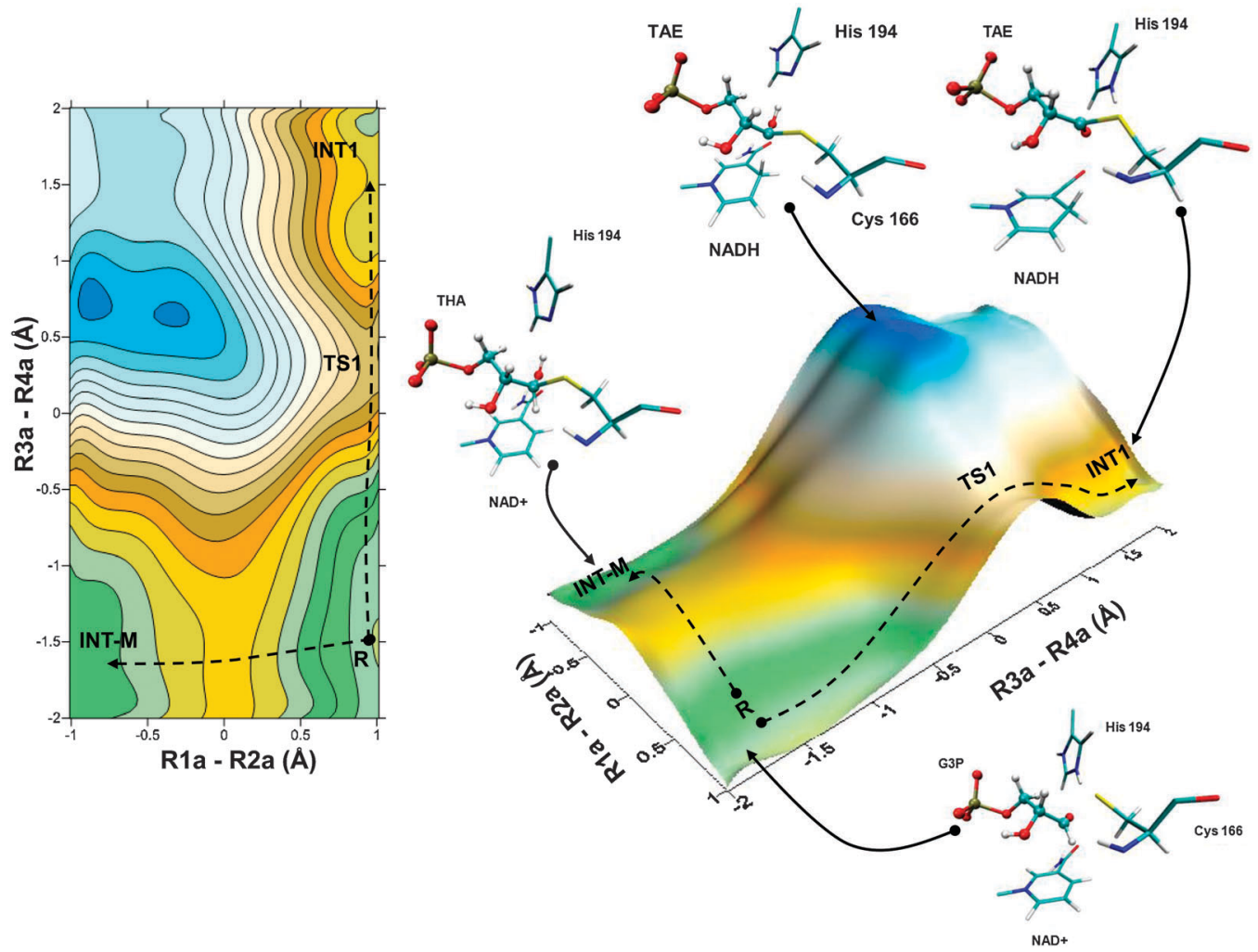

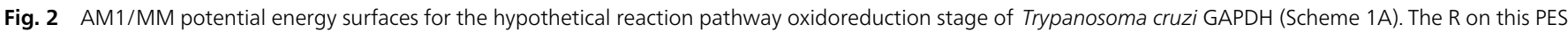

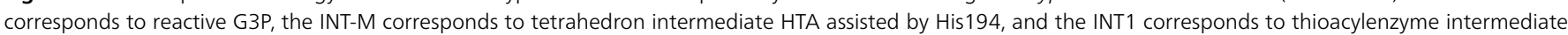
and NADH. 
A

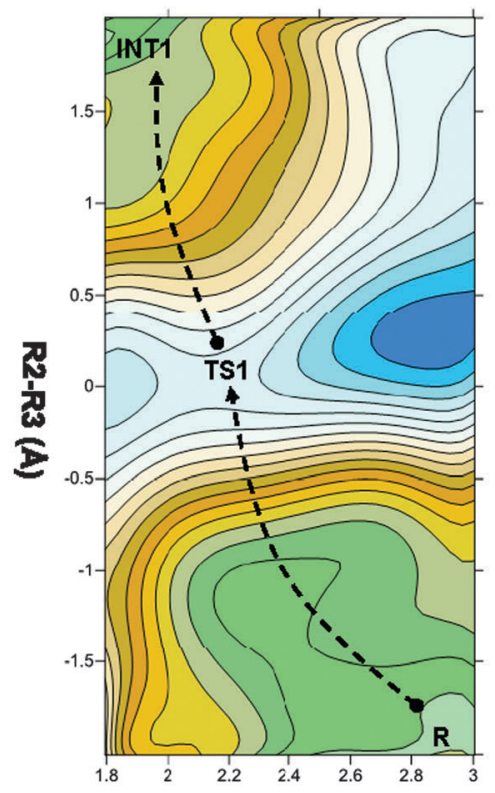

R1 (Á)

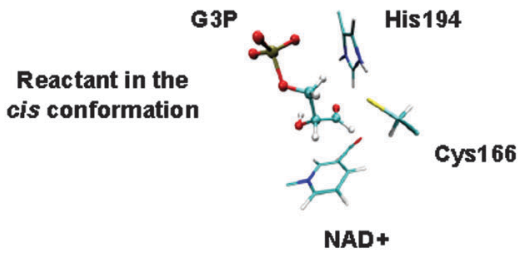

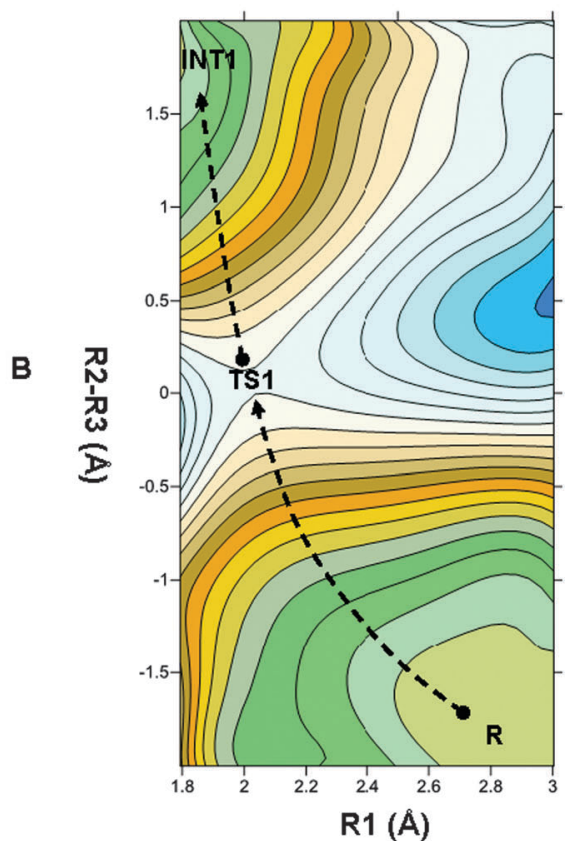

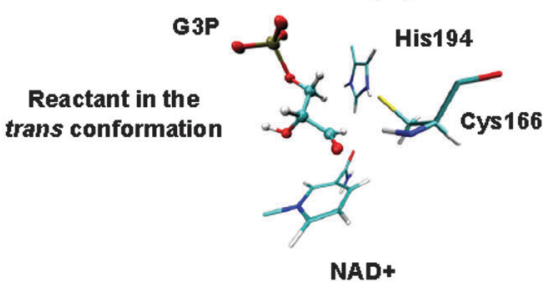

Fig. 3 Two AM1/MM potential energy surfaces obtained with the C3 phosphate first interact with the Pi site and represented two possible conformations (cis and trans for G3P, respectively (A and B), corresponding to the oxidoreduction stage of Trypanosoma cruzi GAPDH).

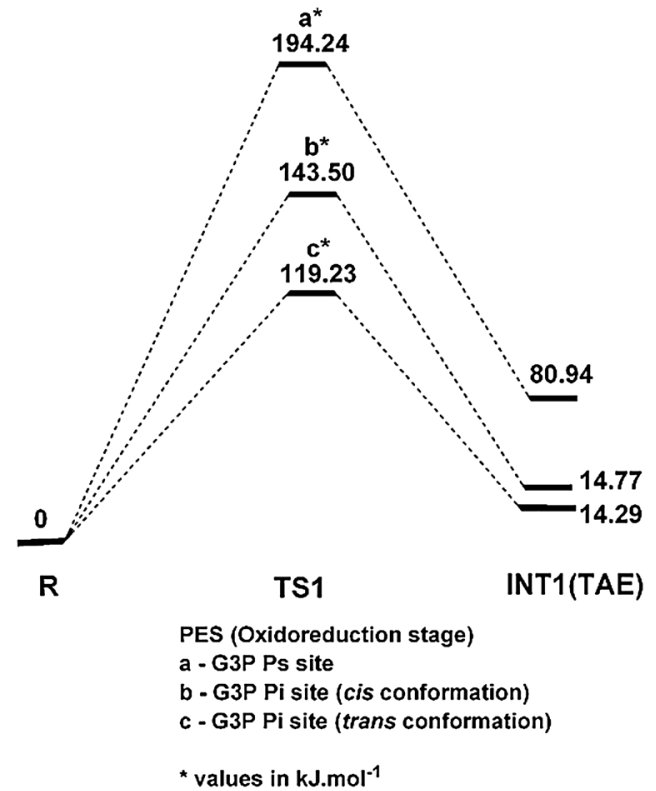

Fig. $4 \mathrm{AM} 1 / \mathrm{MM}$ energy diagrams $\mathrm{a}, \mathrm{b}$ and $\mathrm{c}$ for mechanisms proposed for formation of the thioacylenzyme (TAE): (a) G3P in the Ps site; (b) G3P in the Pi site (cis conformation); (c) G3P in the Pi site (trans conformation).

not lead to intermediate INT1 as expected in the reaction. These conclusions are in agreement with previous studies that point to the Pi site as the first site of interaction of the substrate $\mathrm{G} 3 \mathrm{P},{ }^{18-20}$ in trans conformation. ${ }^{24}$

\section{Free-energy profiles for substrate G3P (first stage)}

Castilho et al. $(2003)^{23}$ proposed that (i) G3P binds to the Pi binding site, positioning the carbonyl group to interact with the positively charged nicotinamidium ring; (ii) hydride transfer to a cofactor leads to a loss of charge interaction and displacement of the reduced cofactor and (iii) the thioacyl intermediate flips to the Ps binding site, and a new NAD+ molecule is now stabilised through hydrogen bonding of the phosphate to ribose and hydrophobic contacts. In this proposed mechanism, the first step of the reaction starts with the attack of Cys $166^{-}$on the $\mathrm{C} 1$ aldehydic group of G3P. Therefore, we have used the Pi binding site where the phosphate moiety of G3P interacts with residues Arg249, Thr167, Ser247 and Thr226 (Fig. 1A) as a starting point to explore the attack of Cys $166^{-}$on the $\mathrm{C} 1$ aldehydic group and the hydride transfer from G3P to NAD+. The values free-energy changes and barriers of the oxidoreduction mechanisms of GAPDH depicted in Fig. 5 can be used to build the free-energy diagram shown in Fig. 6 . The first 2D PMF (Fig. 5A) was obtained using AM1/MM methods through symmetric and anti-symmetric combinations of R1 and R2-R3 (see Scheme 2), respectively. The R1 corresponds to the nucleophilic attack of $\mathrm{Cys} 166^{-}$on the C1 aldehydic group of G3P 
A

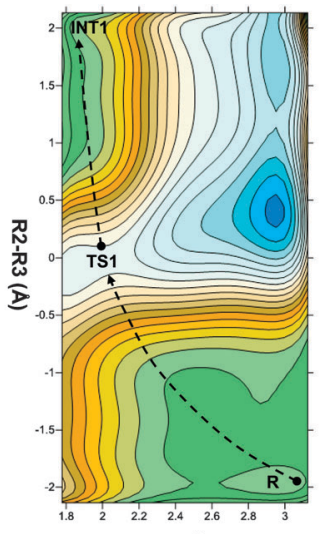

R1 (A)

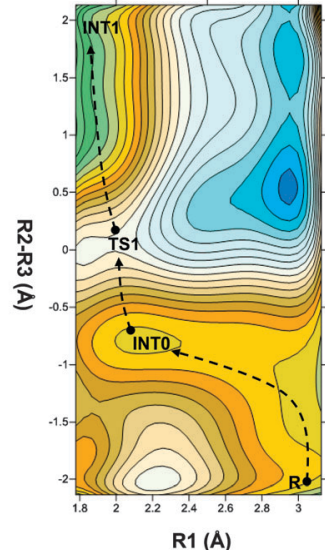

Fig. $52 \mathrm{D}$ PMF corresponding to the oxidoreduction stage of Trypanosoma cruzi GAPDH. Fig. 5A was obtained using AM1/MM, and Fig. 5B was obtained using MP2/6-31G**/MM

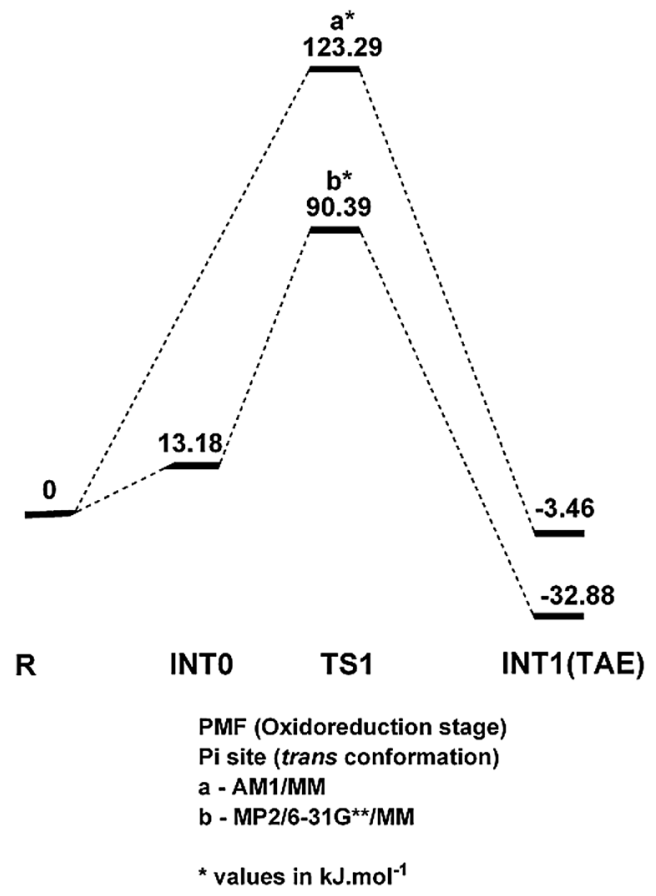

Fig. 6 Free-energy diagrams for formation of the thioacylenzyme (TAE) in the Pi site (trans conformation). (a) Obtained at the AM1/MM level; (b) obtained at the MP2/6-31G**/MM level.

(trans conformation), R2-R3 corresponds to hydride transfer from G3P to NAD+, as described in the section above. The product of the reaction, the INT1 thioester intermediate on the 2D-PMF presented in Fig. 5A, can be obtained via a hydride ion transfer from $\mathrm{C} 1$ of $\mathrm{G} 3 \mathrm{P}$ to NAD+. In the TS structure, TS1 on the 2D-PMF presented in Fig. 5A, the bond distance for $\mathrm{R} 1$ corresponds to $1.92 \pm 0.13 \AA$ indicating advanced progress of the nucleophilic attack of $\mathrm{Cys} 166^{-}$on the C1. On the other hand, the bond distance for R2 in the TS1 corresponds to $1.32 \pm 0.01 \AA$, while R3 corresponds to $1.43 \pm 0.09 \AA$, indicating that hydride transfer from G3P to NAD+ was initiated;
Table 1 Averaged distances of key distances for R, TS1, INT1 structures (in Å). Standard deviations computed during the last 10 ps are reported in brackets (in $\AA$ ) from AM1/MM 2D-PMF

\begin{tabular}{llll}
\hline$\AA$ & R & TS1 & INT1 \\
\hline R1 & $3.34(0.05)$ & $1.92(0.13)$ & $1.77(0.08)$ \\
R2 & $1.09(0.10)$ & $1.32(0.01)$ & $2.32(0.03)$ \\
R3 & $3.94(0.07)$ & $1.43(0.09)$ & $1.08(0.05)$ \\
\hline
\end{tabular}

Table 2 Relative Free Energies for R, TS1 and INT1 structures obtained from the 2D-PMF(AM1/MM). Values are reported in $\mathrm{kJ} \mathrm{mol}^{-1}$

\begin{tabular}{llll}
\hline $\mathrm{kJ} \mathrm{mol}^{-1}$ & $\mathbf{R}$ & TS1 & INT1 \\
\hline & 0 & 123.29 & -3.46 \\
\hline
\end{tabular}

therefore, we can conclude that hydride transfer from G3P to $\mathrm{NAD}+$ occurs after the nucleophilic attack of Cys166 ${ }^{-}$on the C1. Other average key distances obtained from the AM1/MM 2D-PMF are showed in Table 1. The free-energy barrier between $\mathrm{R}$ and TS1, calculated using the AM1 semiempirical level in the $\mathrm{QM}$ region is $123.29 \mathrm{~kJ} \mathrm{~mol}^{-1}$. The Relative Free Energies found for stationary states to the profile traced with the AM1/MM level are provided in Table 2. Fig. 5B shows the second 2D-PMF obtained using MP2/6-31G**/MM methods, with respect to the same reaction coordinates described above and the key averaged distances are provided in Table 3 . The minimum energy pathway is shown by the dashed line in Fig. 5B, where the formation of the hemithioacetal anion intermediate (INT0) after the nucleophile sulfur attack $\mathrm{C} 1$ of the substrate can be observed. In fact, Baer, Brinkman and Brauman (1991) ${ }^{68}$ suggest that tetrahedral adducts of this type are often minima on the reaction potential surface.

Table 4 shows the Relative Free Energies obtained for the oxireduction stage including $2 \mathrm{D}$ interpolated corrections calculated at the MP2/6-31G**. The barrier from this INT0 to TS1 involves hydride transfer from G3P to NAD+ corresponding to $77.21 \mathrm{~kJ} \mathrm{~mol}^{-1}$. These results are consistent with the experimental data of Yun et al. (2000) ${ }^{24}$ and Castilho et al. (2003), ${ }^{23}$ who proposed the formation of an anion hemithioacetal intermediate and thioacylenzyme intermediate. Recently, the

Table 3 Averaged distances of key distances for R, INT0, TS1, INT1 structures (in $\AA$ ). Standard deviations computed during the last 10 ps are reported in brackets (in $\AA$ ) from 2D-PMF (MP2/6-31G**/MM)

\begin{tabular}{lllll}
\hline$\AA$ & R & INT0 & TS1 & INT1 \\
\hline R1 & $3.34(0.05)$ & $2.37(0.04)$ & $1.93(0.13)$ & $1.77(0.08)$ \\
R2 & $1.09(0.10)$ & $1.21(0.08)$ & $1.32(0.01)$ & $2.32(0.03)$ \\
R3 & $3.94(0.07)$ & $2.03(0.62)$ & $1.43(0.09)$ & $1.08(0.05)$
\end{tabular}

Table 4 Relative Free Energies for R, INT0, TS1 and INT1 structures obtained from the 2D-PMF (MP2/6-31G**/MM). Values are reported in $\mathrm{kJ} \mathrm{mol}^{-1}$

\begin{tabular}{lllll}
\hline $\mathrm{kJ} \mathrm{mol}^{-1}$ & $\mathbf{R}$ & INT0 & TS1 & INT1 \\
\hline 0 & 13.18 & 90.39 & -32.88
\end{tabular}


crystal structure of the thioacylenzyme intermediate was obtained by diffusion of the natural substrate within the crystal of the holoenzyme in the absence of inorganic phosphate by Moniot et al. (2008), this evidence supports the results of PMFs.

\section{Free-energy profiles for substrate G3P (second stage)}

The second stage of the proposed catalytic mechanism used by GAPDH begins with the coenzyme exchange step, which could result from a conformational isomerization of the thioacylenzyme intermediate of the C3P of GAP from the Pi to Ps site. Then, the free Pi site of GAPDH could bind inorganic phosphate. ${ }^{23}$ The molecular structure of all protonation states of pyrophosphate has been described by Colvin, Evleth, and Akacem (1995). ${ }^{69}$ In aqueous solution at physiological $\mathrm{pH}(\sim 7.4)$, orthophosphate exists largely as the deprotonated ions $\mathrm{HPO}_{4}{ }^{2-}$. Therefore, we have used $\mathrm{HPO}_{4}{ }^{2-}$ as a starting point to study the second stage of the mechanism used by GAPDH.

Experimental data have shown that the second stage of the catalytic mechanism used by GAPDH takes place in the Ps site. ${ }^{18-20,23,24}$ In this report, we have used the thioacylenzyme intermediate obtained from 2D-PMF as a starting point of the reaction in the Ps site. After the Pi-Ps exchange and assigning the Pi protonation state, a PMF (Fig. 7) was obtained using AM1/MM methods through anti-symmetric combinations of R4-R5 (see Scheme 2) coordinates, which correspond to phosphorolysis of the resulting thioester through the nucleophilic attack of inorganic phosphate on the carbonyl group of the thioacylenzyme (Fig. 7). The product of the reaction is 1,3biphosphoglycerate, $\mathrm{P}$ on the PMF (Fig. 7), completing the reaction cycle. In the TS structure, TS2 (Fig. 7), the bond distance for R5 corresponds to $1.82 \AA$ indicating advanced progress of the nucleophilic attack of the phosphate ion on the $\mathrm{C} 1$, while the bond distance for R4 in the TS corresponds to $1.89 \AA$ indicating bond breaking between C1 and S of Cys166 (Table 5). The energy barrier between INT1 and TS2, calculated
Table 5 Averaged distances of key distances for INT1, TS2, P structures (in Å). Standard deviations computed during the last 10 ps are reported in brackets (in $\AA$ ) from AM1/MM 1D-PMF

\begin{tabular}{llll}
\hline & INT1 & TS2 & P \\
\hline R4 & $1.77(0.08)$ & $1.89(0.12)$ & $3.10(0.10)$ \\
R5 & $2.68(0.13)$ & $1.82(0.05)$ & $1.32(0.08)$
\end{tabular}

using the AM1 semiempirical level in the QM region is $81.81 \mathrm{~kJ} \mathrm{~mol}^{-1}$.

The proposed mechanism of GAPDH T. cruzi for oxidative phosphorylation substrate G3P is represented by snapshots that were obtained on the PMFs are shown in Fig. 8.

\section{Free-energy profiles for a G3P analogue}

Recently, GAPDH analogues have been described as irreversible inhibitors that are covalently bound at the active cysteine residue. ${ }^{23}$ The formation of a thioacyl enzyme analogue obtained through the reaction between the active site cysteine residue and an activated carbonyl group of the G3P analogue leads to an irreversibly bound and stable complex. ${ }^{23}$ In order to understand the mechanism of GAPDH inhibition, we carried out free-energy profiles for a G3P analogue (3-( $p$-nitrophenoxycarboxyl)-3-ethylene propyl dihydroxyphosphonate). We have used the Pi binding site (R in Fig. 9) as a starting point to explore the attack of Cys166 ${ }^{-}$on the carbonyl group of the analogue G3P. A PMF (Fig. 10) was obtained using AM1/MM methods through anti-symmetric combinations of R4R5. The energy profiles along this reaction coordinate correspond to the nucleophilic attack of Cys166 ${ }^{-}$on the atom C1 of the carbonyl group of the G3P analogue (3c). In the TS structure, TS in Fig. 9, the bond distance for R4 corresponds to 1.93 A indicating advanced progress of the nucleophilic attack of Cys166 ${ }^{-}$on the $\mathrm{C} 1$. The energy barrier between R and TS in Fig. 10, calculated using the AM1 semiempirical level in the QM region is $96.23 \mathrm{~kJ} \mathrm{~mol}^{-1}$. This barrier is significantly lower than observed for the substrate (123.29 $\mathrm{kJ} \mathrm{mol}^{-1}$ ), which explains the inhibition mechanism of GAPDH by the G3P analogue inhibitor.

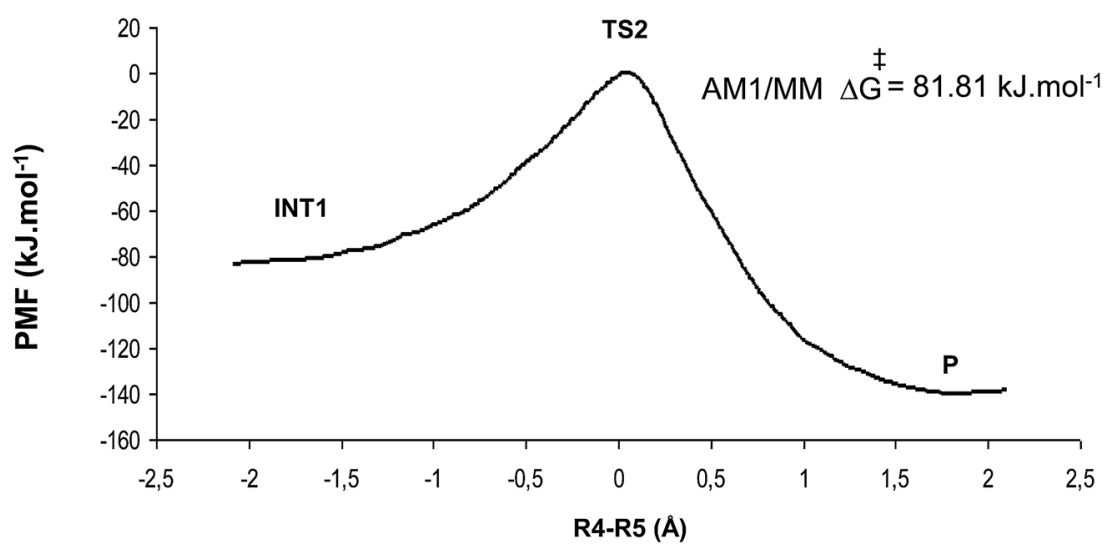

Fig. 7 Plot AM1/MM energies for INT1, TS2 and P structures obtained from the PMFs for corresponding phosphorylation stage of Trypanosoma cruzi GAPDH. Values are reported in $\mathrm{kJ} \mathrm{mol}^{-1}$. 


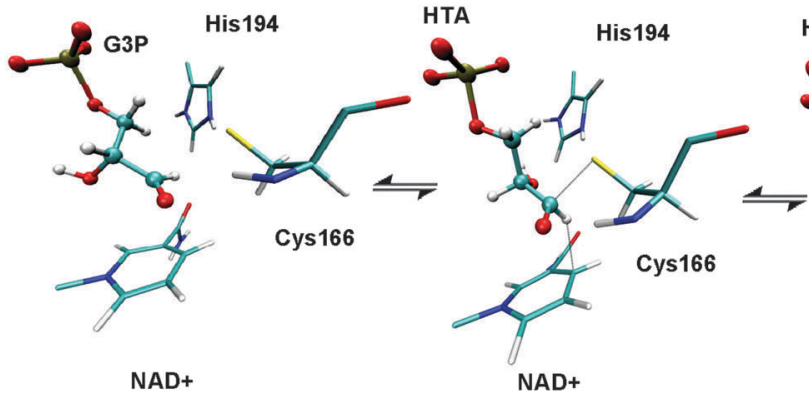

NAD+
NAD+

INTO

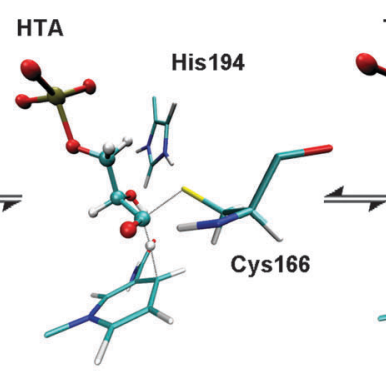

NAD+

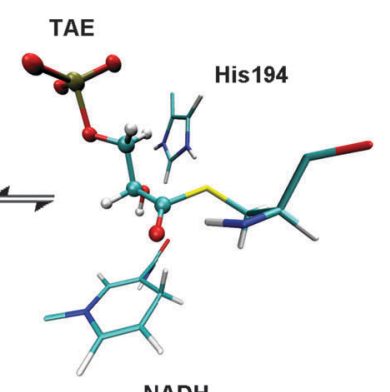

NADH<smiles></smiles>

TS1

INT1
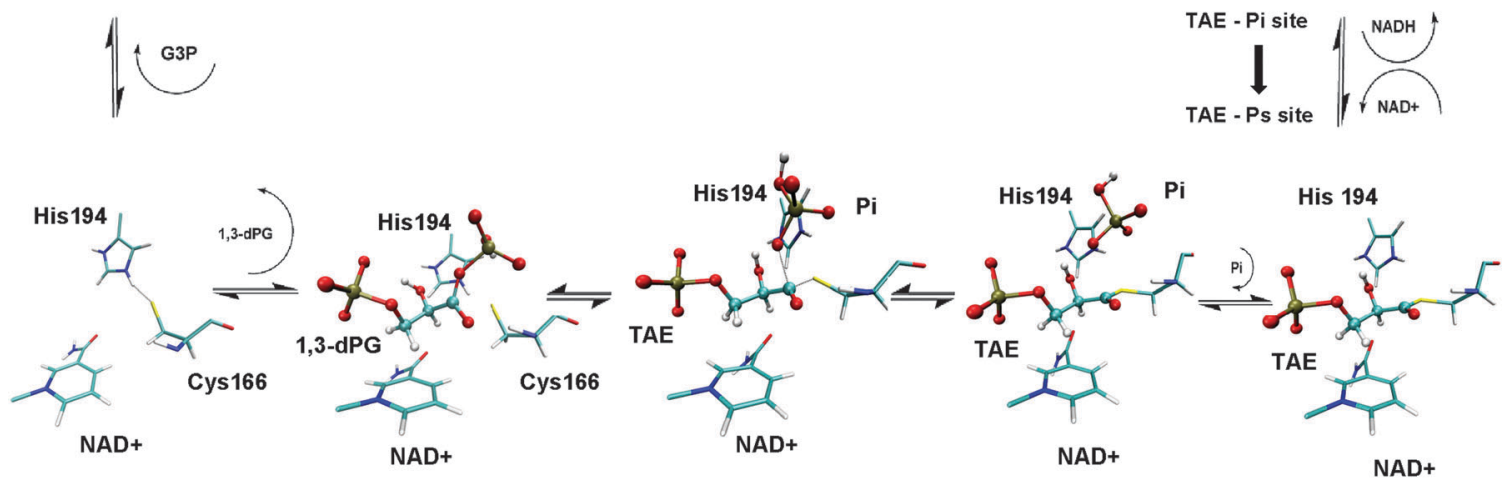

HS

P

TS2

INT1

INT1 - Ps site

Fig. 8 Proposed catalytic mechanism obtained from theoretical calculations.

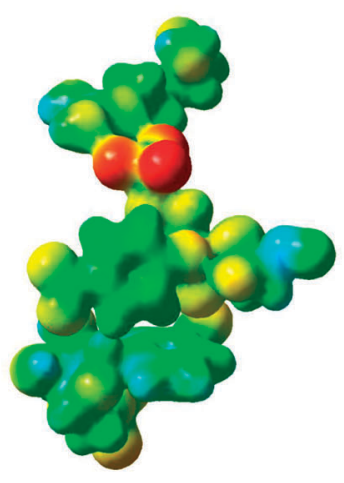

$\mathbf{R}$

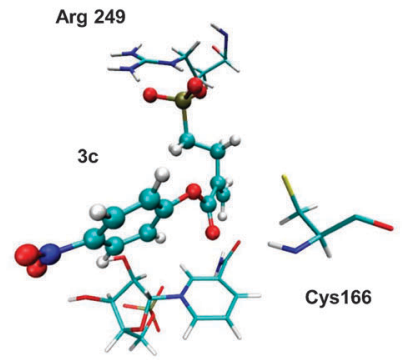

NAD+

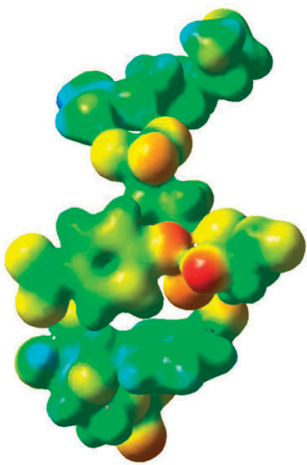

TS

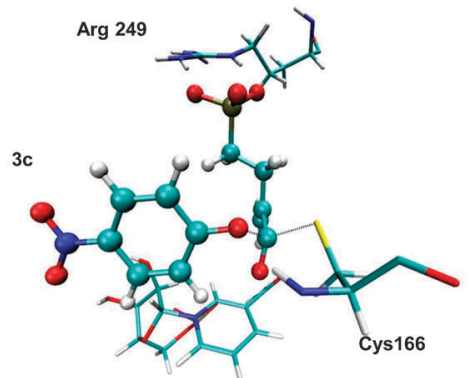

NAD+

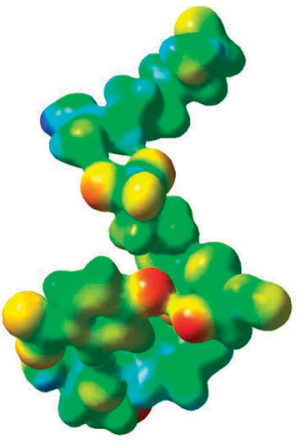

$\mathbf{P}$

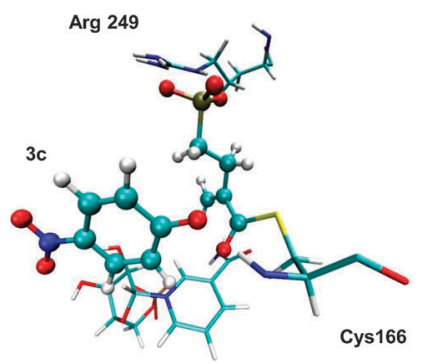

NAD+

3c : 3-(p-Nitrophenoxycarboxyl)-3-ethylene propyl dihydroxyphosphonate

Fig. 9 Map electrostatic potential (MEP) for a G3P analogue (3c). Surfaces derived from B3LYP/6-31G* calculations for the R, TS and P. The increase of negative charges goes from blue (positive) to red (negative). 


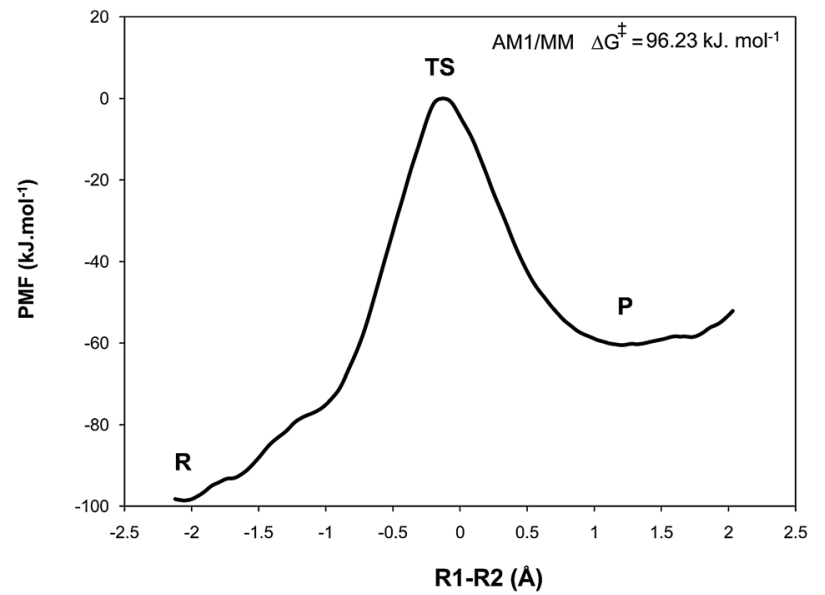

Fig. 10 Free-energy profiles for a G3P analogue [3-( $p$-nitrophenoxycarboxyl)-3ethylene propyl dihydroxyphosphonate] were obtained using AM1/MM. Values are reported in $\mathrm{kJ} \mathrm{mol}^{-1}$.

\section{Interaction energy}

In order to gain insight into the protein-substrate interactions, 100 ps of AM1/MM MD were run for all substrate-protein complexes appearing along the free energy profile (R, INT0, TS1, INT1) constraining the corresponding distinguished reaction coordinate. Averaged protein-substrate interaction energies by residues are displayed in Fig. 11, where positive values correspond to unfavourable interactions while negative values mean stabilisation interactions. Analysis of the results reveals that Arg249 has a significant effect on the ability of the enzyme to bind the substrate/inhibitor, this confirms the important role of the Arg residue of the catalytic site of GAPDH in the stabilisation of negative charges of the C3P group of glyceraldehyde-3-phosphate. This result could explain the marked decrease in the rate of the first steps of the reaction, which lead to acylenzyme formation when the Arg is replaced by Leu as described in the mutagenesis studies of Corbier et al. (1994). ${ }^{20}$ Other important residues interacting with R-protein, INT0protein, TS1-protein and INT1-protein complexes are NAD+, Cys166, Glu109, Thr226, Ser247 and Thr167. The NAD+, Cys166 and Arg249 were proposed to stabilise the tetrahedron intermediate and transition state formed during the reaction. ${ }^{23}$ The weak interactions with His194 observed in the R-protein, INT0protein, TS1-protein and INT1-protein complexes suggest that the role of His194 is not to stabilise the tetrahedron transition state or intermediate formed, but could be limited to activating the nucleophilicity of Cys166. The stability of possible INT0 could be explained by electrostatic interactions with key residues in the active site, such as Cys166 and Thr167. The pattern of interaction of the INT1-protein is close to the TS1-protein but with a weak interaction of $\mathrm{NAD}+$ for $\mathrm{NADH}$. This loss of interaction is important for displacement of the reduced cofactor. $^{23}$ In addition, the interaction with Arg249 is weaker in the INT1-protein complex, which could be necessary for the conformational change from the Pi to Ps site. Finally, the Asp210

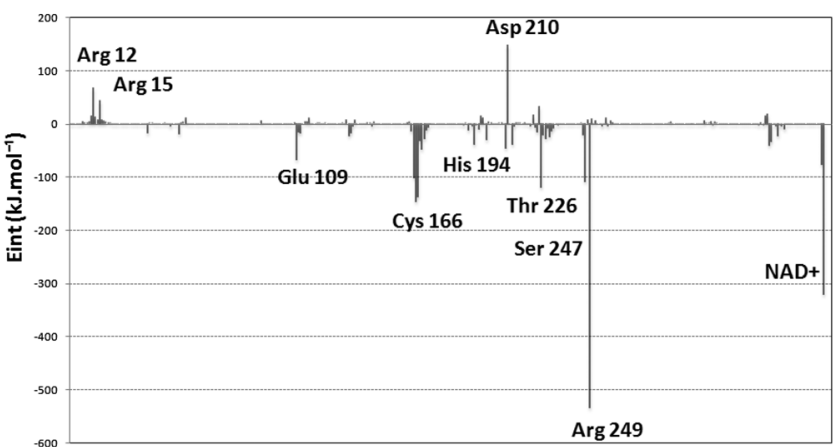

R - Pi Site

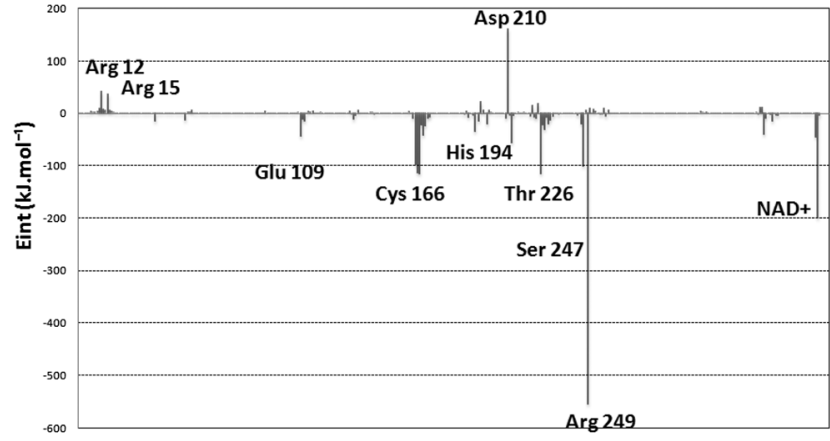

TS1

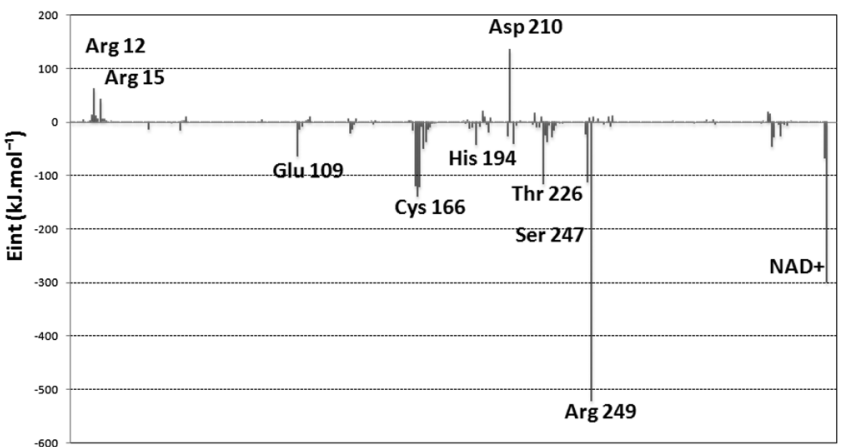

INTO

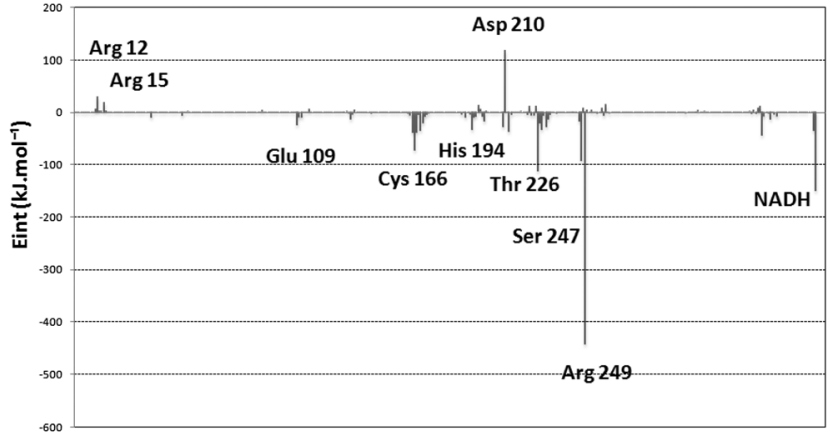

INT1 - Pi Site

Fig. 11 Contributions of individual amino acids to substrate-protein interaction energies (in kJ mol${ }^{-1}$ ) computed for R, INT0, TS1 and INT1. 

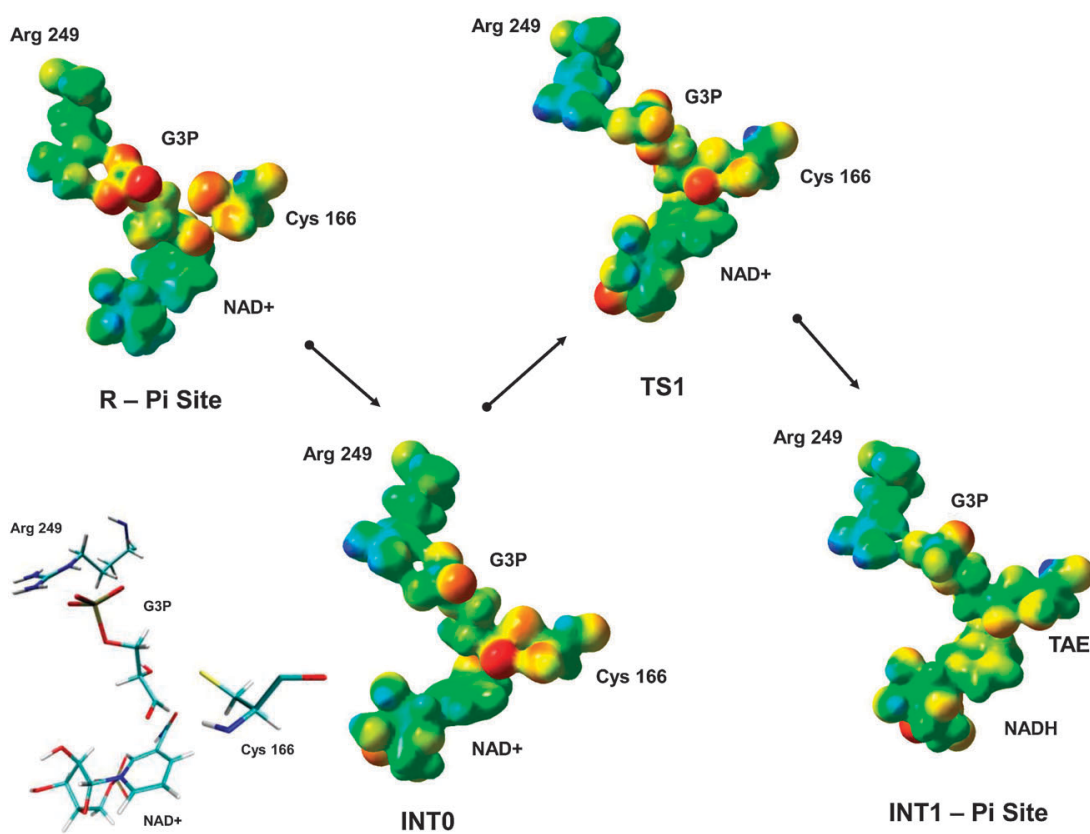

INT1 - Pi Site

Fig. 12 Map electrostatic potential (MEP) surfaces derived from B3LYP/6-31G* calculations for R, INT0, TS1 and INT1. The increase of negative charges goes from blue (positive) to red (negative).

has an unfavourable interaction with G3P, which is negatively charged; on the other hand, Asp210 stabilises Arg249 through a bridge salt in the absence of the substrate, ${ }^{47}$ which could be important in the release of the reaction product.

\section{Molecular electrostatic potential (MEP)}

Fig. 9 and 12 show the MEP surfaces derived from B3LYP/3$21 \mathrm{G}(\mathrm{d})$ using Gaussian calculations (Frisch, 2004) ${ }^{70}$ and GaussView $^{71}$ for the $3 \mathrm{c}$ inhibitor and substrate G3P, where the nucleophilic regions (negative electronic potential) are shown in red and the electrophilic regions (positive electrostatic potential) are shown in blue. These surfaces correspond to an isodensity value of 0.02 au. The reactive (R) displays large negative regions around the $\mathrm{C} 3 \mathrm{P}$ group matching the position of Arg249 and Cys166 around the S atom, which is ready to attack the $\mathrm{C} 1$ atom of G3P. The oxyanion at thiohemiacetal C1 of G3P (INT0) interacts with the $\mathrm{NH}$ of Cys166, which is consistent with the distance observed between C1 of G3P and $\mathrm{N}$ of Cys166 of $2.98 \AA$ in $E$. coli. ${ }^{24}$ In addition, the tetrahedral transition state (TS1) contains a large negative charge around the oxygen at the $\mathrm{C} 1$ of G3P and could interact more tightly with $\mathrm{NH}$ of Cys166 and Thr167 and the C2 hydroxyl group of G3P; this points to the oxygen of the carboxamide group of the nicotinamide ring that through a hydrogen interaction helps stabilise the transition state, therefore, keeping the G3P in an energetically more favourable trans conformation, as proposed by Yun et al. (2000). ${ }^{24}$ The R, INT0, TS1 and INT1 structures show that the nicotinamide ring becomes more negatively charged during the reaction. This ring is more negative in the INT1 structure, which suggests that hydride transfer to NAD+ leads to a loss of charge. The MEP of INT1 closely resembles the transition state (TS1) showing the formation of an oxoanion in this enzymatic reaction. Analysis of MEP (Fig. 9) reveals that the $3 \mathrm{c}$ inhibitor is likely the substrate (R) and displays large negative (red) regions around the $\mathrm{C} 3 \mathrm{P}$ group matching the position of Arg249. In the TS and P structure of the $3 \mathrm{c}$ inhibitor, a large negative region on Cys166 around the $\mathrm{S}$ atom is also observed.

\section{Conclusions}

We have investigated the catalytic mechanism used by GAPDH involving the G3P substrate from 2D-PMF surfaces obtained through QM/MM MD calculations. The results show that the first stage of the reaction is not assisted by His194 and takes place in the Pi site. Our results reveal that the reaction may start with the attack of Cys $166^{-}$on the $\mathrm{C} 1$ atom of the substrate, which leads to an intermediate similar to the intermediate anion hemithioacetal (HTA) described by Yun et al. $(2000)^{24}$ and Castilho et al. (2003). ${ }^{23}$ The second stage corresponds to phosphorolysis via nucleophilic attack of the inorganic phosphate on the carbonyl group of the thioacyl enzyme. These theoretical results are consistent with the flip-flop model for this enzyme mechanism proposed from the experimental results. In accordance with results of interaction energy by residues, Arg249 has a crucial role in the ability of the enzyme to bind the substrate, which interacts also with NAD+, Cys166, Glu109, Ser247, Thr167 and Thr226 in the active site of GAPDH. The MEP reveals a large negative region around the C3P group matching the position of Arg249, which is consistent with the results of interaction energies computed by residues. Finally, the inhibition mechanism of GAPDH by the $3 \mathrm{c}$ inhibitor reveals that the barrier for the inhibition reaction is lower than that 
observed for a natural substrate. This result may explain the potential of this inhibitor.

\section{Acknowledgements}

We thank the Spanish Ministry Ministerio de Ciencia e Inovación for Project CTQ2009-14541-C02-01/02, Universitat Jaume I-Bancaixa Foundation for Project P1 1A2010-08, Generalitat Valenciana for projects ACOMP/2012/119 and ACOMP/2012/243, GV/2012/044 and Prometeo/2009/053, and SEUI for financial support of a Hispano-Brasileño collaboration project (PHB2005-0091-PC). The authors also acknowledge the Servei d'Informatica, Universitat Jaume I for generous allotment of computer time. M.R. thanks CAPES for their financial support and the warm hospitality during the research stay at the Departament de Química Física i Analítica, Universitat Jaume I.

\section{References}

1 C. A. Horae and F. G. Wallace, Nature, 1966, 244, 69.

2 http://www.dndi.org/diseases/chagas.html.

3 L. V. Kirchhoff, New Engl. J. Med., 1993, 329, 639.

4 F. R. Opperdoes and P. Borst, FEBS Lett., 1977, 80, 360.

5 F. R. Opperdoes, Annu. Rev. Microbiol., 1987, 41, 127.

6 J. C. Engel, B. M. Franke de Cazzulo, A. O. Stoppani, J. J. Cannata and J. J. Cazzulo, Mol. Biochem. Parasitol., 1987, 26, 1.

7 B. M. Bakker, P. A. M. Michels, F. R. Opperdoes and H. V. Westerhoff, J. Biol. Chem., 1999, 274, 14551.

8 B. M. Bakker, H. V. Westerhoff, F. R. Opperdoes and P. A. M. Michels, Mol. Biochem. Parasitol., 2000, 106, 1.

9 H. L. Segal and P. D. Boyer, J. Biol. Chem., 1953, 204, 265.

10 D. R. Trentham, Biochem. J., 1971, 122, 5.

11 D. R. Trentham, Biochem. J., 1971, $122,71$.

12 P. J. Harrigan and D. R. Trentham, Biochem. J., 1973, 135, 695.

13 P. J. Harrigan and D. R. Trentham, Biochem. J., 1974, 143, 353.

14 R. G. Duggleby and D. T. Denis, J. Biol. Chem., 1974, 249, 167.

15 D. Moras, K. W. Olsen, M. N. Sabesan, M. Buehner, G. C. Ford and M. G. Rossmann, J. Biol. Chem., 1975, 250, 9137.

16 J. I. Harris and M. Waters, in The Enzymes, ed. P. D. Boyer, Academic Press, New York, 3rd edn, 1976, ch. 13.

17 A. Soukri, A. Mougin, C. Corbier, A. Wonacott, C. Branlant and G. Branlant, Biochemistry, 1989, 28, 2586.

18 T. Skarzynski, P. C. Moody and A. J. Wonacott, J. Mol. Biol., 1987, 193, 171.

19 S. Michels, E. Rogalska and G. Branlant, Eur. J. Biochem., 1996, 235, 641.

20 C. Corbier, S. Michels, A. J. Wonacott and G. Branlant, Biochemistry, 1994, 33, 3260.

21 F. Talfournier, N. Colloc'h, J. P. Mornon and G. Branlant, Eur. J. Biochem., 1998, 252, 447.
22 S. Moniot, S. Bruno, C. Vonrhein, C. Didierjean, S. Boshi-Muller, M. Vas, G. Bricogne, G. Branlant, A. Mozzarelli and C. Corbier, J. Biol. Chem., 2008, 21693.

23 M. S. Castilho, F. Pavão, G. Oliva, S. Ladame, M. Willson and J. Périe, Biochemistry, 2003, 42, 7143.

24 M. Yun, C. G. Park, J. Y. Kim and H. W. Park, Biochemistry, 2000, 39, 10702.

25 I. Korndorfer, B. Steipe, R. Huber, A. Tomschy and R. Jaenicke, J. Mol. Biol., 1995, 246, 511.

26 H. Kim, I. K. Feil, C. L. Verlinde, P. H. Petra and W. G. Hol, Biochemistry, 1995, 34, 14975.

27 F. M. Vellieux, J. Hajdu and W. G. Hol, Acta Crystallogr., Sect. D: Biol. Crystallogr., 1995, 51, 575.

28 S. Ladame, M. S. Castilho, C. H. T. P. Silva, C. Denier, V. Hannaert, J. Périe, G. Oliva and M. Willson, Eur. J. Biochem., 2003, 279, 4564.

29 S. Martí, J. Andres, V. Moliner, E. Silla, I. Tuñón, J. Bertrán and M. F. Field, J. Am. Chem. Soc., 2001, 123, 1709.

30 J. Lameira, C. N. Alves, I. Tuñón, S. Martí and V. Moliner, J. Phys. Chem. B, 2011, 115, 6764.

31 C. N. Alves, S. Martí, R. Castillo, J. Andrés, V. Moliner, I. Tuñòn and E. Silla, Chem.-Eur. J., 2007, 13, 7715.

32 A. Thomas and M. J. Field, J. Am. Chem. Soc., 2002, 124, 12432.

33 N. Kanaan, S. Martí and V. Moliner, J. Phys. Chem. A, 2009, 113, 2176.

34 A. Warshel and J. Levitt, Mol. Biol., 1976, 103, 227.

35 M. J. Field, P. A. Bash and M. Karplus, J. Comput. Chem., 1990, 11, 700.

36 S. Martí, J. Andrés, V. Moliner, E. Silla, I. Tuñón and J. Bertran, Chem. Soc. Rev., 2008, 37, 2634.

37 S. Martí, M. Roca, J. Andrés, V. Moliner, E. Silla, I. Tuñón and J. Bertrán, Chem. Soc. Rev., 2004, 33, 98.

38 H. M. Senn and W. Thiel, Angew. Chem., Int. Ed., 2009, 48, 1198.

39 H. G. Schlegel, J. Comput. Chem., 2003, 24, 1514.

40 A. S. Carneiro, J. Lameira and C. N. Alves, Chem. Phys. Lett., 2011, 514, 336.

41 J. L. Jenkis and J. J. Tanner, Acta Crystallogr., Sect. D: Biol. Crystallogr., 2006, 62, 290.

42 H. C. Watson, E. Duee and W. D. Mercer, Nat. New Biol., 1972, 240, 130.

43 J. J. Tanner, R. M. Hecht and K. L. Krause, Biochemistry, 1996, 35, 2597.

44 E. Duee, L. Olivier-Deyris, E. Fanchon, C. Corbier, G. Branlant and O. Dideberg, J. Mol. Biol., 1996, 257, 814.

45 H. Kim and W. G. Hol, J. Mol. Biol., 1998, 278, 5.

46 F. M. Vellieux, J. Hajdu, C. L. Verlinde, H. Groendijk, R. J. Read, T. J. Greenhough, J. W. Campbell, K. H. Kalk, J. A. Littlechild and H. C. Watson, Proc. Natl. Acad. Sci. U. S. A., 1993, 90, 2355.

47 D. H. Souza, R. C. Garratt, A. P. Araujo, B. G. Guimaraes, W. D. Jesus, P. A. Michels, V. Hannaert and G. Oliva, FEBS Lett., 1998, 424, 131.

48 F. Pavão, M. S. Castilho, M. T. Pupo, R. L. A. Dias, J. B. Correa, J. B. Fernandes, M. F. G. F. da Silva, 
J. Mafezoli, P. C. Vieira and G. Oliva, FEBS Lett., 2002, 520, 13.

49 M. R. Murthy, R. M. Garavito, J. E. Johnson and M. G. Rossmann, J. Mol. Biol., 1980, 138, 859.

50 T. Skarzynski and A. J. Wonacott, J. Mol. Biol., 1988, 203, 1097.

51 M. J. Field, M. Albe, C. Bret, F. Proust-de Martin and A. Thomas, J. Comput. Chem., 2000, 21, 1088.

52 M. J. Field, A practical Introduction to the Simulation of Molecular Systems, Cambridge University Press, Cambridge, UK, 1999.

53 J. Antosiewicz, J. A. McCammon and M. K. Gilson, J. Mol. Biol., 1994, 238, 415.

54 L. Hui, A. D. Robertson and J. H. Jensen, Proteins, 2005, 61, 704 .

55 A. Cartier, D. Brown, B. Maigret, S. Boschi-Muller, S. RahuelClermont and G. Branlant, Theor. Chem. Acc., 1999, 101, 241.

56 R. H. Byrd, P. Lu, J. Nocedal and C. Zhu, J. Sci. Comput., 1995, 16, 1190.

57 M. J. S. Dewar, E. G. Zoebisch, E. F. Healy and J. J. P. Stewart, J. Am. Chem. Soc., 1985, 107, 3902.

58 W. L. Jorgensen, D. S. Maxwell and J. Tirado-Rives, J. Am. Chem. Soc., 1996, 118, 11225.

59 W. L. Jorgensen, J. Chandrasekhar, J. D. Madura, R. W. Impey and M. L. Klein, J. Chem. Phys., 1983, 79, 926.

60 J. G. Kirkwood, J. Chem. Phys., 1935, 3, 300.

61 D. A. McQuarrie, Statistical Mechanics, Harper \& Row, New York, 1976, pp. 266.
62 B. Roux, Comput. Phys. Commun., 1995, 91, 275.

63 G. M. Torrie and J. P. Valleau, J. Comput. Phys., 1977, 23, 187.

64 G. K. Shenter, B. C. Garret and D. G. Truhlar, J. Chem. Phys, 2003, 119, 5828.

65 M. Roca, V. Moliner, J. J. Ruiz-Pernía, E. Silla and I. Tuñón, J. Phys. Chem. A, 2006, 110, 503.

66 L. Polgar, Eur. J. Biochem., 1975, 51, 63.

67 C. Didierjean, C. Corbier, M. Fatih, F. Favier, S. BoschiMuller, G. Branlant and A. Aubry, J. Biol. Chem., 2003, 278, 12968.

68 S. Baer, E. A. Brinkman and J. I. Brauman, J. Am. Chem. Soc., 1991, 113, 805.

69 E. M. Colvin, E. Evleth and Y. Akacem, J. Am. Chem. Soc., 1995, 117, 4357.

70 M. J. Frisch, Gaussian 03, revision C.02, Gaussian, Inc., Wallingford, CT, 2004.

71 R. Dennington, T. Keith, J. Millam, K. Eppinnett, W. L. Hovell and R. Gilliland, GaussView, V3.0, Semichem Inc., Shawnee Mission, KS, 2003.

72 J. J. Ruiz-Pernía, E. Silla, I. Tuñón and S. Martí, J. Phys. Chem. B, 2006, 110, 17663.

73 M. W. Schmidt, K. K. Baldridge, J. A. Boatz, S. T. Elbert, M. S. Gordon, J. H. Jensen, S. Koseki, N. Matsunaga, K. A. Nguyen, S. J. Su, T. L. Windus, M. Dupuis and J. A. Montgomery, J. Comput. Chem., 1993, 14, 1347. GAMESS Version 11, $2008 \mathrm{R} 1$. 